\title{
Involvement of the Nerve Fibers in the Human Skin
}

\section{Neurofibromatosis}

Tammaro Antonella ${ }^{1}$, Cavallotti Claudia ${ }^{2}$, Parisella Francesca Romana ${ }^{3}$, Giulianelli Veronica ${ }^{1}$, Gabriella De Marco $^{1}$, Elena Pacella ${ }^{4}$, Fernanda Pacella ${ }^{4}$, Persechino Severino ${ }^{1}$ and Cavallotti Carlo ${ }^{5}$

1. Department of Dermatology, Sapienza University, Rome 00189, Italy

2. Department of Histopathology, San Gallicano Hospital, Rome 00153, Italy

3. Premedical School of Medicine, University of Towson, Maryland 21252-0001, USA

4. Department of ophthalmology, Policlinico Umberto I, Sapienza University, Rome 0016, Italy

5. Department of Human Anatomy, Sapienza University, Rome 00161, Italy

Received: August 21, 2013 / Accepted: January 11, 2015 / Published: January 30, 2015.

\begin{abstract}
Neurofibromatosis type 2 is a well known disease of the human skin. Its microscopic and ultramicroscopic features are also well defined. Aim: The aim of this work was to study the involvement of catecholaminergic nerve fibers in the human skin neurofibromatosis. Bioptic fragments of the human skin have been harvested from healthy and diseased subjects. On these specimens the following analysis were performed: 1) light microscopic observation after colouring with hematoxyline-Eosine. 2) Immunochemical staining for Protein Gene Product 9.5. 3) Fluorescent staining for catecholaminergic nerve fibers. 4) Quantitative analysis of images by means of the Quantimet analyzer Leica. 5) Statistical analysis of the quantitative morphological data comparing the healthy with diseased subjects. Comparing the light microscopy images in normal and pathological subjects the authors can affirm that the skin neurofibromatosis induces a strong decrease of the nerve fibers cutted in small pieces and destroyed. Also the catecholaminergic nerve fibers are strongly reduced and destroyed. Quantitative analysis of images and statistical analysis of the morphological data confirm that neurofibromatosis induces strong changes of the skin nerve fibers. The authors' results confirm that the neurofibromatosis type 2 induces an almost total destruction of the skin nerve fibers.
\end{abstract}

Key words: Neurofibromatosis, humans, skin nerve fibers, cathecolaminergic fibers.

\section{Introduction}

The multiple neurofibromatosis was first described by Recklinghausen [1]. In the next years many clinical and morphological studies were performed on this disease. Chino and Tsuruhara with the aid of electron microscopy described the ultrastructural aspects of the involved tissues [2]. Poirier and coworkers studied the ultrastructural and nosologic relations of the neurofibromas [3]. Pennelli and Stoppelli demonstrated some anatomo-clinical aspects of vesicle neurofibromatosis [4]. Holm and coworkers

Corresponding author: Tammaro Antonella, M.D., Ph.D., research fields: dermatology, anatomopathology, allergology. E-mail: tammaroantonella@gmail.com. first studied multiple cutaneous neuromas showing that the skin lesions are formed by the typical coffee and milk spots, pathognomonic of the disease [5]. When they are six or more and with a diameter of 1.5 $\mathrm{cm}$ (already found at the birth or during the first year of life).

Microscopical study of these spots shows melanocyte with giant melanosomas, circular bodies of 500 Angstrom in diameter, freckles and scattered freckles, white nevi [5]. Moreover in skin neurofibromas Schochet and Barret [6] demonstrated the presence of aberrant tactile corpuscles.

The neurofibromatosis can become worse and change in tumor [7]. Lassmann and coworkers found a reaction of connective tissue fibers in the tumor 
neurofibromatosis [8]. In the year after Lassmann and coworkers performed some electron microscopic and autoradiografic studies on cutaneous neurofibromas [9]. Kamata by mean of ultrastructural studies on neurofibromatosis demonstrated that acetylcholinesterase activity can be involved in this disease [10]. Kao and coworkers described a solitary neurilemmoma in the cutaneous nerve fibers [11].

Peripheral neurofibromatosis can involve also the lumbosacral nerves as described by Bequet and coworkers [12]. Donner and coworkers found neural sheath tumors in many major nerves of the human body [13]. Also Goujon and coworkers studied the association neurinomas-neurobifromas [14].

Finally Drouet and coworkers performed an appraisal of the association neurofibromatosis-neuropathies [15].

In the present work the authors will investigate the involvement of the catecholaminergic nerve fibers in the human skin neurofibromatosis.

\section{Materials and Methods}

From the archive of the authors' hospital the authors used the photographic documentation that refers to a case of cutaneous peripheral neurofibromatosis. Moreover in the last five years there have been performed at San Gallicano Hospital three skin biopsies to confirm the suspected diagnosis of cutaneous peripheral neurofibromatosis. After careful observation of the microscopic slices diagnosis was confirmed in only one case, while the other two cases were found to be healthy.

Fragments of skin from the three biopsies were used for further morphological examinations after obtaining the informed consent of patients.

The fragments of skin taken from fibromatosis nodules or from healthy subjects were studied for the following investigations:

- Basic histological examination for detecting the morphological details;

- Immunochemical staining for PGP (protein gene product) 9.5 to detect almost all of the nerve fibers present in the samples;

- Histochemical staining for the catecholaminergic nerve fibers to highlight the possible implications of the sympathetic nervous system (catecholaminergic) in cutaneous peripheral neurofibromatosis;

- QAI (quantitative analysis of images) for to receive quantitative data from the morphological images;

- Statistical analysis of the data comparing normal and pathological values.

Here is a short description of the methods that the authors used: all the experiments that the authors performed were first approved by the local Ethical Committee, and were conducted in accordance with the Declaration of Helsinki in its revised edition, the Guidelines of Good Clinical Practice (CMP/ICH/135/95), and international and local regulatory requirements. For each case a report was prepared, indicating the age and sex of patients as well as general informations. The morphological units knew only the anonymous number of each sample. Therefore, a "double blind" procedure was used in all experiments.

The fresh material was fixed, dehydrated, paraffin-embedded, cut in serial sections (8-10 micrometers thick) on a rotative microtome. The sections were deposed on glass slices and processed for hematoxylin and eosin staining to assess general microanatomy and to detect morphometric parameters.

Other sections were stained for the immunohistochemical detection of nerve fiber profiles using as general marker for nervous tissue the Protein Gene Product 9.5.

For the staining of CNF (catecholaminergic nerve fibers) a glyoxylic acid-induced fluorescence technique was used. Briefly, immediately before use the staining solution was prepared by adding a solution of $0,236 \mathrm{M}$ potassium phosphate (pH 7.4) 0,2 $\mathrm{M}$ sucrose and $1 \%$ glyoxylic acid. This staining is named SPG (sucrose, phosphate and glyoxylic acid). 
The slides with samples were immediately dipped in this solution for $5 \mathrm{~min}$. to assure a comparable fluorescence it is important to standardize times and temperatures without intervals.

After staining, the sections were drained, covered with non-auto-fluorescent immersion oil, heated at $95{ }^{\circ} \mathrm{C}$ for $5 \mathrm{~min}$ and observed. In fact, the sections were immediately observed and photographed to prevent any diffusion and photodecomposition of the fluorescence. The sections were examined and photographed under a Zeiss photomicroscope equipped with exciter and barrier filters and with a mercury lamp for observation of fluorescence.

The a-specific fluorescence was barred with special filters, furnished as an optional by Zeiss. Photographs were submitted to the quantitative analysis of fluorescent structures using a Quantimet 2000 image Analyzer (Leitz) equipped with specific software. The following parameters were measured 1) total area of fluorescent structures. 2) number of nervous varicosities. 3) number of crossing or intersections of nerve fibers. All these values are expressed in C.U. (conventional units) furnished directly by QAI.

Statistical analysis: owing to the rather limited number of the subjects ( 3 in total) it is impossible a statistical analysis. Nevertheless the authors can compare the values obtained in normal subjects with those of the diseased patient.

\section{Results}

Photographic documentation of a case of skin neurofibromatosis in reported in Fig. 1. As can be seen the dorsal lumbar region of the patient shows numerous little spots coffee-milk and many neurofibromas of the skin. Fig. 2 is a high magnification of the boxed area of the previous figure. The authors can see a portion of wrinkled skin with numerous neurofibromas. Both these photos came from the photographic archive of the hospital.

The authors' experimental results are reports in the next Figs. 3-7. Fig. 3 is a light microscopic observation of the human skin in a patient affected by neurofibromatosis type 2 . The authors can observe numerous polinucleated cells and some giant-cells. These microscopic features are patognomonic for the diagnosis of skin neurofibromatosis.

Fig. 4 is a light microscopic observation of the normal human skin coloured for PGP 9.5. this immunohistochimical staining is a general marker for nervous tissue. The deeper and the upper nervous plexuses of the skin are well defined and well coloured.

Fig. 5 on the contrary is a light microscopic image of the skin stained for PGP 9.5 of a subject affected by skin neurofibromatosis. Comparing Fig. 4 with Fig. 5 the authors can see a strong decrease of the nerve fibers cutted in a small pieces and destroyed in the pathologic skin. A slice of normal human skin stained for catecholaminergic nerve fibers is reported in Fig. 6 as fluorescent light microscopy. The authors can observe the deeper and the upper cutaneous nerve plexuses.

Fig. 7 is a fluorescent light microscopic image of a slice of human skin coming from a patient affected by cutaneous neurofibromatosis type 2. Comparing Fig. 6 with Fig. 7 the authors can observe a strong decrease of the catecholaminergic nerve fibers cut in small pieces and destroyed as the authors find in pathologic case.

\section{Discussion}

Neurofibromatosis is a disease that is divided into type $1 \mathrm{NF}$ and type $2 \mathrm{NF}$. Both types are genetic in origin, however type $1 \mathrm{NF}$ and type $2 \mathrm{NF}$ involve mutation in different genes [16]. Type 1NF (von Recklinghausen's disease) is the more common form of NF accounting approximately $90 \%$ of reported cases with a prevalence of one case in 3000 birth. Type $1 \mathrm{NF}$ is found in three forms, peripheral, central and visceral [17]. The peripheral form is characterized by multiple cutaneous coffee-milk spots that are associated with multiple cutaneous nerve sheath 


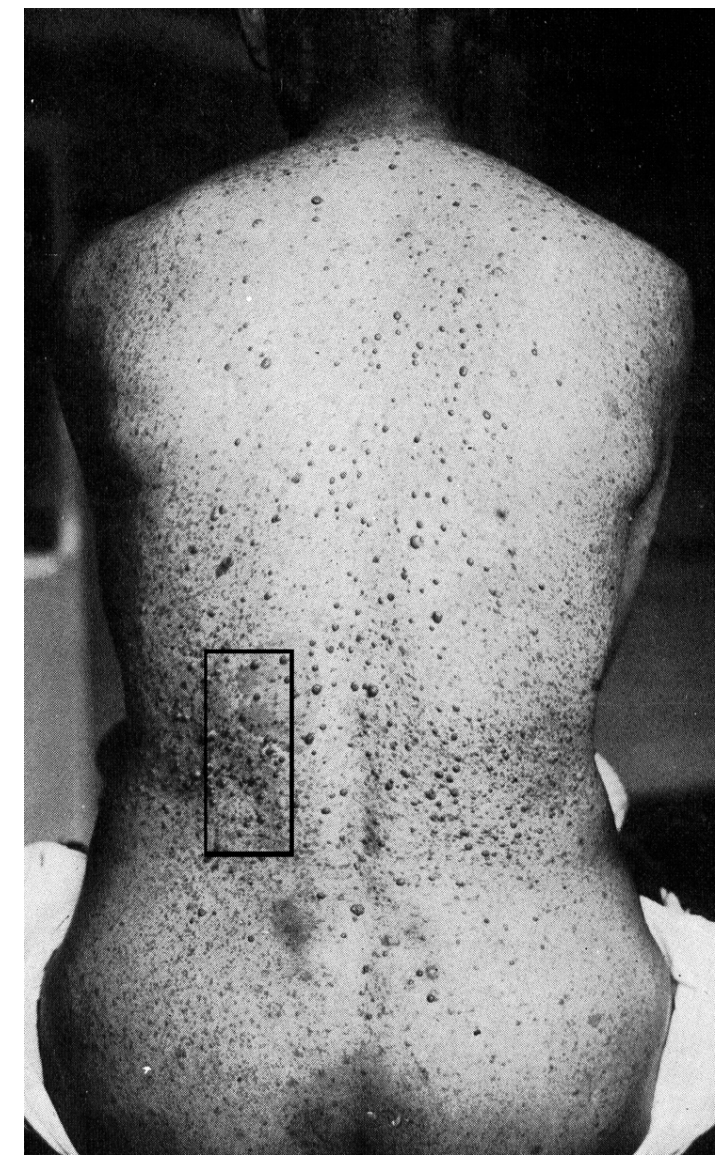

Fig. 1 Picture of the dorsal lumbar region of a patient with neurofibromatosis type 2 . It can be seen that observe many neurofibromas and numerous dark spots. Boxed area reported at high magnification in the Fig 2.

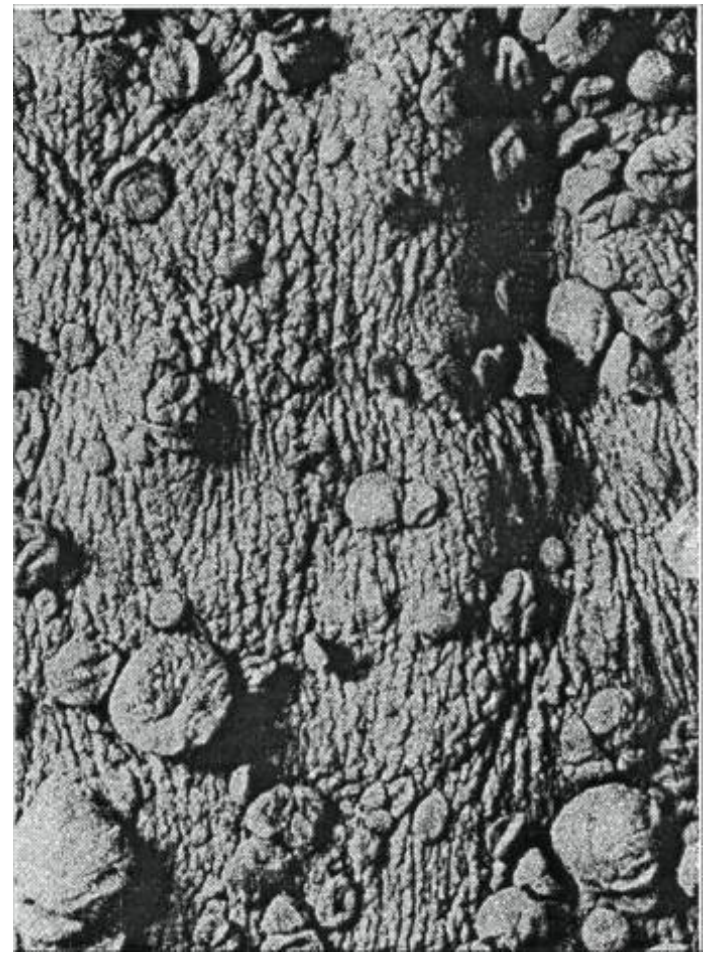

Fig. 2 Boxed area of Fig 1 at high magnification $(25 \mathrm{x})$. It can be seen the numerous neurofibromas of the skin. 


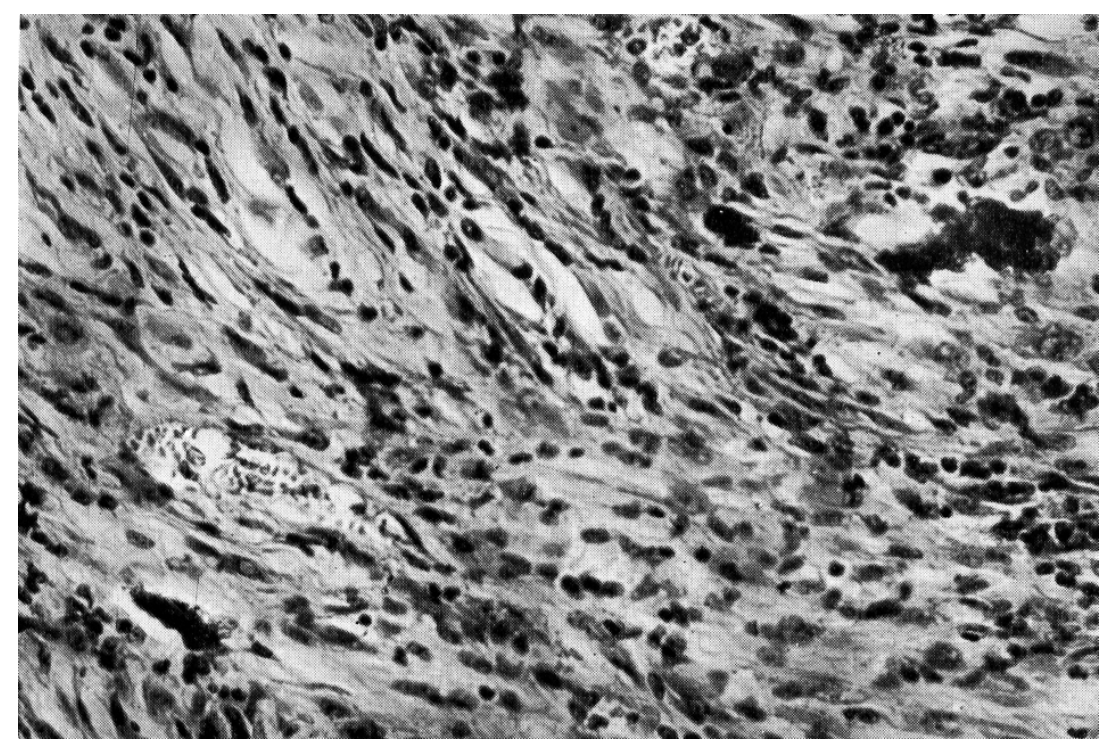

Fig. 3 Light microscopy image of a neurofibromas of the skin. It can be observed numerous polinucleated and giant cells (magnification 400x). From the collection of Dermatologic Hospital S.Andrea Rome.

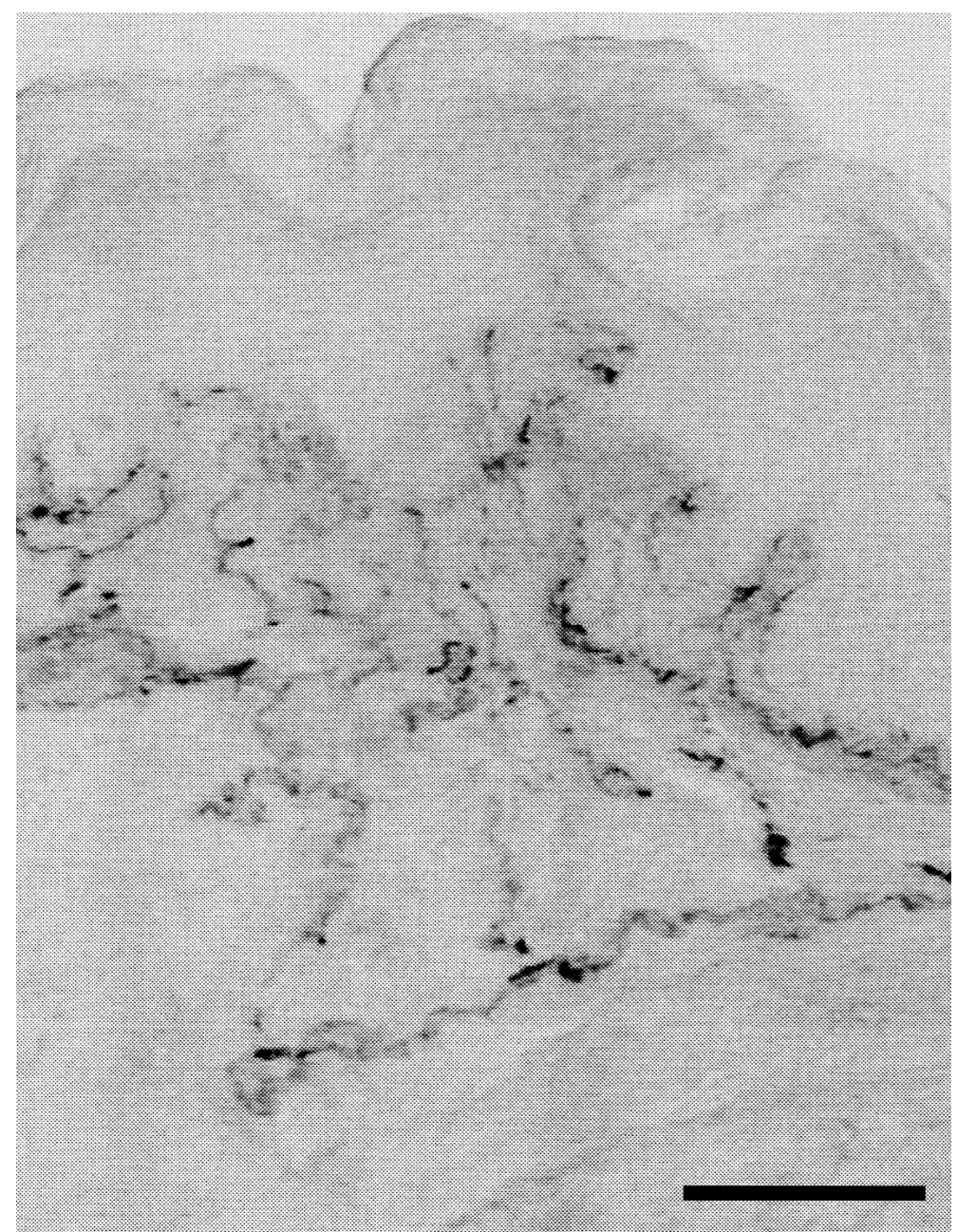

Fig. 4 Light microscopy image of the human normal skin. It can be observed the upper and the deeper nerve plexuses coloured for PGP 9.5. Calibration bar 40micrometres. 


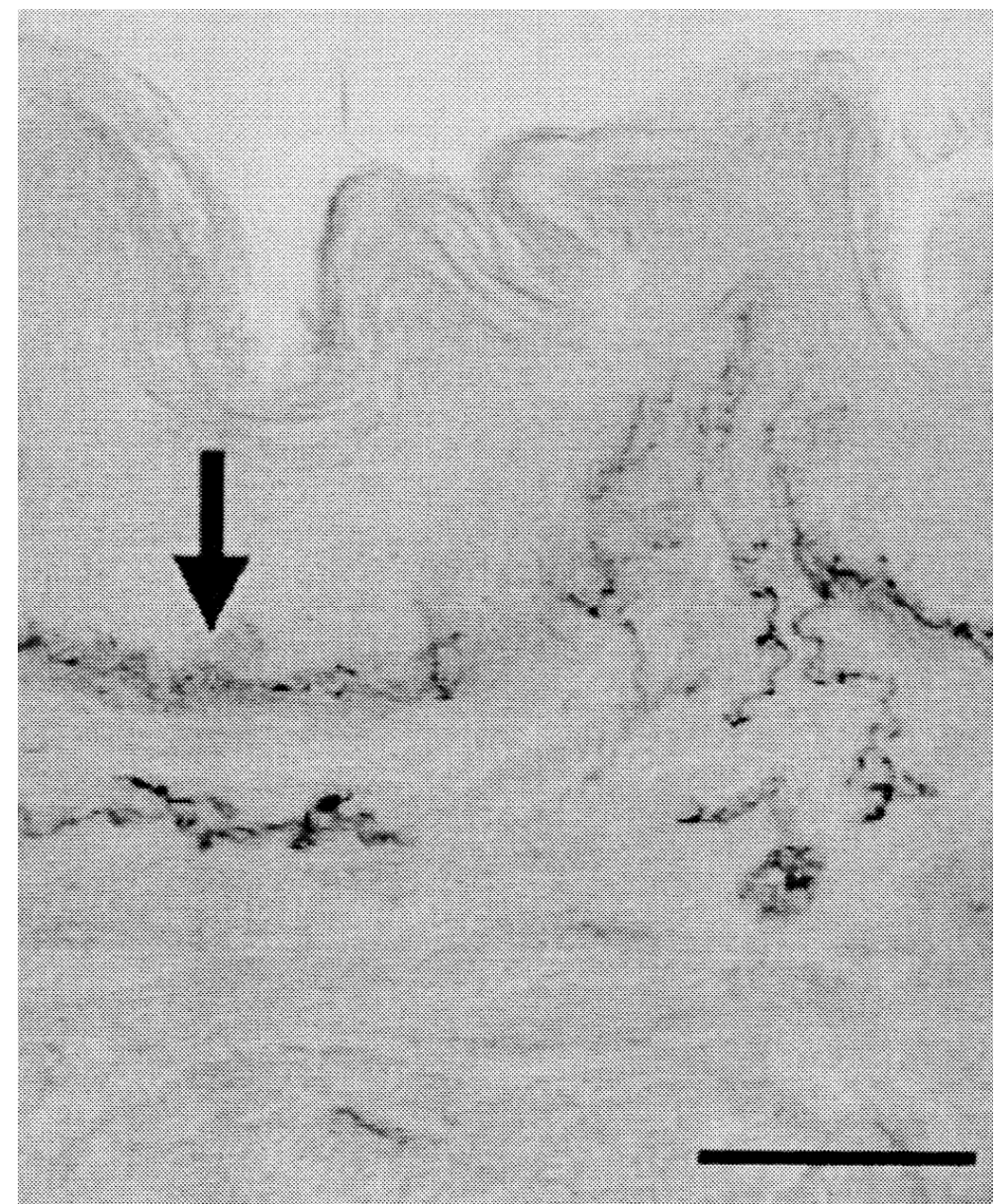

Fig. 5 Light microscopy image of the human skin of a patient affected by Neurofibromatosis type 2. Same colouring and magnification of Fig 4. It can be seen that a strong decrease of the nerve fibers, cutted in small pieces and destroyed.

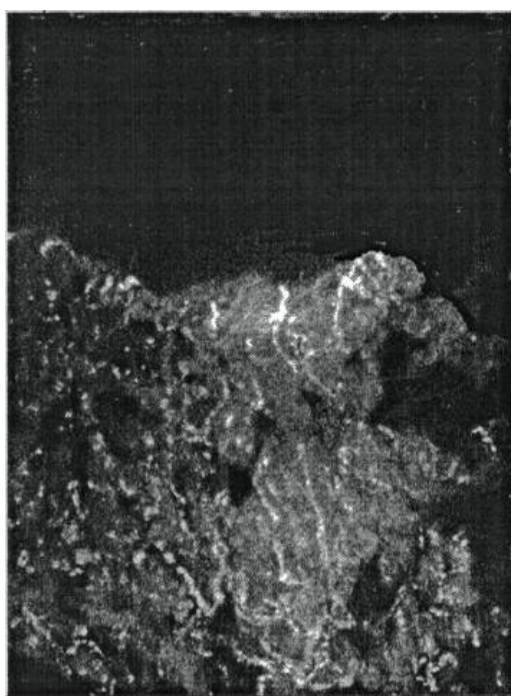

Fig. 6 Fluorescent light microscopy of the human normal skin. It can be observed the upper and the deeper cutaneous nerve plexuses stained for catecholaminergic nerve fibers. Calibration bar 40 micrometers.

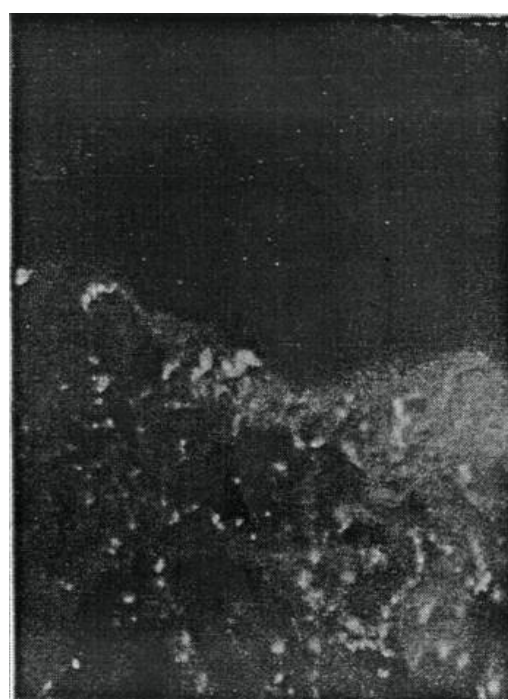

Fig. 7 Fluorescent light microscopy microscopy of the human skin of a patient affected by neurofibromatosis type 2 . It can be observed a strong decrease of the catecholaminergic nerve fiberscutted in small pieces and destroyed. Calibration bar $\mathbf{4 0}$ micrometers. 
tumors. The central form shows multiple intracranial and intravertebral tumors arising from the central nervous system and cranial and spinal nerve roots. The visceral form involves ganglioneuromas of the autonomic nervous system in the viscera. NF1 is further associated with attention-deficit hyperactivity disorder and autism spectrum disorder [18]. Type 2NF generally involves the development of schwannomas, meningomas and ependynomas However the tumors in type $2 \mathrm{NF}$ can be malignant and extremely aggressive when it involves mesothelioma [19].

Langerhans in 1868 described the presence of cutaneous nerve fibers intraepithelial highlighted through techniques of impregnation with heavy metals. Intraepithelial cutaneous nerve fibers were rare and difficult to highlight, that is why he thought they were or few or staining artifacts.

In the early 1900s, the presence of cutaneous nerve fibers has been well documented in the dermis, while in skin epithelium the nerve fibers are restricted to the cells of the basal layer.

The nervous system skin was highlighted with great detail when (only around the year 1960) have been used immunohistochemical techniques able to mark each neurotransmitters present in sensory nerve fibers or the fibers of the visceral sympathetic and parasympathetic nervous system, which are distributed to the cutaneous vessels and also the cutaneous sensory corpuscles. The use of immunohistochemical techniques has allowed to establish that the cutaneous nervous system is very developed and includes a deep nerve plexus, located in the deep dermis, and a superficial nerve plexus located in the superficial dermis that gives rise to a dense intraepidermal nerve network. Unfortunately there are no universal markers for all types of cutaneous nerve fibers, but only markers for different types of nerve fibers. Only the sum of all nerve fibers evidenced by all the different markers can gives a complete view of the cutaneous innervation. However, there are some limitations, such as the same nerve fibers can be detected by more than one marker. To date there is still no ubiquitous marker that stains all nerve fibers. For this purpose were used neurofilaments antibodies anti nucleus antibodies such as the NSE (neural specific enolase).

In recent years the marker considered more effective to highlight the most part of the nerve cells is the antibody against the PGP 9.5. The corresponding antigen is present in the cytosol of the nerve cells and therefore the neural anti PGP 9.5 is able to highlight the nerve cell and its extensions and is particularly useful for marking intraepidermal nerve fibers. Recently to determine the degree of impairment of skin sensitivity in peripheral neuropathies has been used a particular neuropathological method, using quantitative analysis of intraepidermal nerve fibers, the so-called index of intraepidermal innervation (IENF $=$ Intraepidermic Nerve Fibers). Skin sensitivity is directly proportional to the amount of superficial skin nerve fibers and especially to the amount of intraepidermal nerve fibers. Using many markers (called functional), the authors can transform the morphological data in functional: therefore the authors can have a matter of clinical interest with which the authors can make the prognosis of many neuropathies of the skin also predicting any eventual functional recovery.

\section{Conclusions}

Neurofibromatosis type 2 is a well known disease of the human skin with well defined microscopic and ultramicroscopic features. It is also characterized by an alteration of skin nerve fibers, that controls cutaneous circulation in physiological conditions. This is a very important function because both deep and superficial circulation provide for the manteinance of the hydration and the proper nutritional supply of the skin and are necessary to remove its catabolites.

The authors' study shows that in human skin neurofibromatosis, there is a severe destruction of cutaneous nerve fibers, especially of the 
catecholaminergic ones. The authors think that these alterations can also influence cutaneous circulation of affected skin, because superficial and deep vessels are regulated by skin nerve fibers. Therefore, nerves damage affects not only cutaneous sensitivity but can also alter the skin integrity, worsening the clinical features in patients affected by NF 2 .

\section{References}

[1] Antônio, J. R., Goloni-Bertollo, E. M., and Arroyo Trídico, L. 2013. "Neurofibromatosis: Chronological History and Current Issues" An Bras Dermatol. 88 (3): 329-343.

[2] Chino, F., and Tsuruhara, T. 1968. "Electron Microscopic Study of von Recklinghausen's Disease." Jpn. J. Med. Sci. Biol. 21 (4): 249-257.

[3] Poirier, J., Escourolle, R., and Castaigne, P. 1968. "Neurofibromas in von Recklinghausen's Disease. Ultrastructural Study and Nosologic Relation to Neurinomas.” Acta Neuropathol. 10 (4): 279-294.

[4] Pennelli, N., and Stoppelli, I. 1968. "Anatomo-Clinical, Histological and Ultrastructural Aspects of Vesical Neurofibromatosis." Tumori. 54 (3): 237-253.

[5] Holm, T. W., Prawer, S. E., Sahl, W. J. Jr., and Bart, B. J. 1973. "Multiple Cutaneous Neuromas." Arch Dermatol. 107 (4): 608-610.

[6] Schochet, S. S. Jr., and Barrett, D. A. 1974. "Neurofibroma with Aberrant Tactile Corpuscles." Acta Neuropathol. 28 (2): 161-165.

[7] Kimura, M., Kamata, Y., Matsumoto, K., and Takaya, H. 1974. "Electron Microscopical Study on the Tumor of von Recklinghausen's Neurofibromatosis." Acta Pathol Jpn. 24 (1): 79-91.

[8] Lassmann, H., Gebhart, W., and Stockinger, L. 1975. "The Reaction of Connective Tissue Fibers in the Tumor of Recklinghausen's Disease." Virchows Arch B Cell Pathol. 19 (2): 167-77.

[9] Lassmann, H., Jurecka, W., and Gebhart, W. 1976. "Some Electron Microscopic and Autoradiographic Results Concerning Cutaneous Neurofibromas in von Recklinghausen's Disease." Arch Dermatol Res. 255 (1): 69-81.
[10] Kamata, Y. 1978. "Study on the Ultrastructure and Acetylcholinesterase Activity in von Recklinghausen's Neurofibromatosis." Acta Pathol Jpn. 28 (3): 393-410.

[11] Kao, G. F., Laskin, W. B., and Olsen, T. G. 1989. "Solitary Cutaneous Plexiform Neurilemmoma (Schwannoma): a Clinicopathologic, Immunohistochemical, and Ultrastructural Study of 11 Cases." Mod Pathol. 2 (1): 20-26.

[12] Béquet, D., Labauge, P., Larroque, P., Renard, J. L., and Goasguen, J. 1990. "Peripheral Neurofibromatosis and Involvement of Lumbosacral Nerves. Value of Imaging." Rev Neurol (Paris). 146 (12): 757-761.

[13] Donner, T. R., Voorhies, R. M., and Kline, D. G. 1994. "Neural Sheath Tumors of Major Nerves." J. Neurosurg. 81 (3): 362-373.

[14] Goujon, J. M., Bataille, B., Menet, E., and Lapierre, F. 1997. "Neurinomas-Neurofibromas." Neurochirurgie. 43 (1): 35-38.

[15] Drouet, A., Wolkenstein, P., Lefaucheur, J. P., Pinson, S., Combemale, P., Gherardi, R. K., Brugières, P., Salama, J., Ehre, P., Decq, P., and Créange, A. 2004. "Neurofibromatosis 1-Associated Neuropathies: a Reappraisal.” Brain. 127 (9): 1993-2009.

[16] Ghalayani, P., Saberi, Z., and Sardari, F. 2012. "Neurofibromatosis Type I (von Recklinghausen's disease): A Family Case Report and Literature Review." Dent Res J. (Isfahan) 9 (4): 483-488.

[17] Protopapas, A., Sotiropoulou, M., Haidopoulos, D., Athanasiou, S., Loutradis, D., and Antsaklis, A. 2011. "Ovarian Neurofibroma: a Rare Visceral Occurrence of Type 1 Neurofibromatosis and an Unusual Cause of Chronic Pelvic Pain” J. Minim Invasive Gynecol. 18 (4): 520-524.

[18] Walsh, K. S., Vélez, J. I., Kardel, P. G., Imas, D. M., Muenke, M., Packer, R. J., Castellanos, F. X., and Acosta, M. T. 2012. "Symptomatology of Autism Spectrum Disorder in a Population with Neurofibromatosis Type 1." Dev Med Child Neurol 55 (2): 131-138.

[19] Beltrami, S., Branchetti, E., Sariyer, I. K., Otte, J., Weaver, M., and Gordon, J. 2013 "Neurofibromatosis Type 2 Tumor Suppressor Protein, NF2, Induces Proteasome-Mediated Degradation of JC Virus T-antigen in Human Glioblastoma." PLoS One 8 (1): e53447. 